\title{
Prediction of clinically relevant hyperkalemia in patients treated with peptide receptor radionuclide therapy
}

Constantin Lapa ${ }^{1 *}{ }^{*}$, Rudolf A Werner ${ }^{1 \dagger}$, Christina Bluemel ${ }^{1}$, Katharina Lueckerath ${ }^{1}$, Dirk O Muegge ${ }^{2}$, Alexander Strate ${ }^{3}$, Heribert Haenscheid ${ }^{1}$, Andreas Schirbel', Martin S Allen-Auerbach ${ }^{4}$, Ralph A Bundschuh ${ }^{5}$, Andreas K Buck ${ }^{1}$ and Ken Herrmann ${ }^{1}$

\begin{abstract}
Background: Peptide receptor radionuclide therapy (PRRT) is applied in patients with advanced neuroendocrine tumors. Co-infused amino acids (AA) should prevent nephrotoxicity. The aims of this study were to correlate the incidence of AA-induced hyperkalemia (HK) $(\geq 5.0 \mathrm{mmol} / \mathrm{l})$ and to identify predictors of AA-induced severe HK (>6.0).

Methods: In 38 patients, standard activity of ${ }^{177}$ Lu-labelled somatostatin analogs was administered. Pre-therapeutic kidney function was assessed by renal scintigraphy and laboratory tests. For kidney protection, AA was co-infused. Biochemical parameters (potassium, glomerular filtration rate, creatinine, blood urea nitrogen (BUN), sodium, phosphate, chloride, and lactate dehydrogenase (LDH)) were obtained prior to 4 and $24 \mathrm{~h}$ after the AA infusion. Incidence of HK $(\geq 5.0)$ was correlated with pre-therapeutic kidney function and serum parameters. Formulas for the prediction of severe HK (>6.0) were computed and prospectively validated.
\end{abstract}

Results: At $4 \mathrm{~h}, \mathrm{HK}(\geq 5.0)$ was present in $94.7 \%$ with severe HK (>6.0) in 36.1\%. Values normalized after $24 \mathrm{~h}$ in $84.2 \%$. Pre-therapeutic kidney function did not correlate with the incidence of severe HK.

Increases in $\mathrm{K}^{+}$were significantly correlated with decreases in phosphate $(r=-0.444, p<0.005)$ and increases in BUN $(r=0.313, p=0.056)$. A baseline BUN of $>28 \mathrm{mg} / \mathrm{dl}$ had a sensitivity of $84.6 \%$ and a specificity of $60.0 \%(\mathrm{AUC}=0.75)$ in predicting severe $\mathrm{HK}$ of $>6.0$ (phosphate, $\mathrm{AUC}=0.37$ ).

Computing of five standard serum parameters (potassium, BUN, sodium, phosphate, LDH) resulted in a sensitivity of $88.9 \%$ and a specificity of $79.3 \%$ for the prediction of severe HK $>6.0$ (accuracy $=81.6 \%$ ).

Conclusions: A combination of serum parameters predicted prospectively the occurrence of relevant HK with an accuracy of $81.6 \%$ underlining its potential utility for identifying 'high-risk' patients prone to PRRT.

Keywords: NET; PRRT; Hyperkalemia; Kidney function; MAG3; Amino acids

\section{Background}

Peptide receptor radionuclide therapy (PRRT) with radiolabelled somatostatin agonists such as ${ }^{90} \mathrm{Y}$ - or ${ }^{177} \mathrm{Lu}$ labelled-[1,4,7,10-tetraazacyclododecane- $\mathrm{NN}^{\prime}, \mathrm{N}^{\prime \prime}, \mathrm{N}^{\prime}$ "-tetraacetic acid]-d-Phe ${ }^{1}, \mathrm{Tyr}^{3}$-octreotoc/-tate) DOTATOC/-TATE (is routinely used for advanced and/or metastatic neuroendocrine tumors (NET) which overexpress somatostatin

\footnotetext{
*Correspondence: lapa_c@ukw.de

${ }^{\dagger}$ Equal contributors

'Department of Nuclear Medicine, University Hospital Würzburg, Oberdürrbacher Str. 6, Würzburg 97080, Germany

Full list of author information is available at the end of the article
}

receptor subtype II (SSTR II) [1-3]. Although the treatment is generally well tolerated, side effects like nausea, myelosuppression, and renal failure have been reported $[4,5]$. Tubular reabsorption and retention of the radiopeptide at the proximal tubule may lead to an excessive radiation dose to the kidney. Therefore, pre-therapeutic assessment of renal function is mandatory and is routinely assessed by means of laboratory tests (creatinine, glomerular filtration rate (GFR), and blood urea nitrogen (BUN)) or nuclear imaging techniques (e.g., renal scintigraphy with ${ }^{99 \mathrm{~m}} \mathrm{Tc}$ mercapto-acetylglycyl-glycyl-glycine [ $\left.\left.{ }^{99 \mathrm{~m}} \mathrm{Tc}-\mathrm{MAG} 3\right]\right)$. 
For kidney protection, various protocols using co-infusion of positively charged amino acids (AA) like L-arginine and L-lysine have been introduced [6-10]. However, side effects including nausea, vomiting, and clinically relevant hyperkalemia have been described $[11,12]$. In a recent study including patients with NET undergoing PRRT with ${ }^{90}$ Y-DOTATOC, Giovacchini et al. reported the incidence of hyperkalemia $(>5.0 \mathrm{mmol} / \mathrm{l})$ in more than three-fourths of patients with potassium levels up to $6.7 \mathrm{mmol} / \mathrm{l}$, thereby raising awareness of this potentially life-threatening adverse effect induced by AAcoinfusion [13].

In clinical routine practice, early or even pre-therapeutic identification of patients at risk for developing severe hyperkalemia (>6.0 $\mathrm{mmol} / \mathrm{l}$ ) due to AA-coinfusion would be of high relevance. Therefore, the aims of this study were to assess the incidence and severity of hyperkalemia $(\geq 5.0 \mathrm{mmol} / \mathrm{l})$ and to identify predictors of PRRT-related severe hyperkalemia (>6.0 $\mathrm{mmol} / \mathrm{l})$.

\section{Methods}

\section{Study design}

The study consists of a retrospective and a prospective part. In the retrospective analysis, 38 consecutive patients were included. Incidence and severity of hyperkalemia ( $\geq 5.0 \mathrm{mmol} / \mathrm{l}$ ) were correlated with pre-therapeutic kidney function. Three formulas for the prediction of severe hyperkalemia $(>6.0 \mathrm{mmol} / \mathrm{l})$ were computed retrospectively from different combinations of serum parameters. Thereafter, these formulas were prospectively validated in a cohort including another 38 patients.

This study comprised of only retrospective analysis of routinely acquired data and therefore the institutional review board (ethics committee of the Medical Faculty of the University of Würzburg) waived the requirement for additional approval. All patients gave written informed consent to receive standard-of-care PRRT. In October 2013, the routinely administered amount of AA was reduced from $75 \mathrm{~g}$ to $50 \mathrm{~g}$ to comply with the most recent version of the joint International Atomic Energy Agency (IAEA), European Association of Nuclear Medicine (EANM), and Society of Nuclear Medicine and Molecular Imaging (SNMMI) practical guidance [6].

\section{Patients}

From July 2013 to December 2013, 38 consecutive patients (24 males, 14 females) referred for PRRT were enrolled. Their mean age was $62 \pm 14$ years (range, 24 to 82 ). The general exclusion criteria, as defined by the joint IAEA, EANM, and SNMMI practical guidance, were applied [6]. All patients had progressive disease. Out of the 38 subjects enrolled, 7 received their first treatment cycle. The following risk factors for the occurrence of kidney toxicity were assessed in all patients: diabetes mellitus $(n=5)$ and arterial hypertension $(n=12)$. Additionally, potassium-sparing anti-hypertensive medications and long-term use of NSAIDs $(n=17)$ were recorded. Pretherapeutic ECG was unremarkable in all patients. None of the subjects had a history of arrhythmogenic diseases.

From January 2014 to April 2014, the formulas for hyperkalemia-risk-assessment derived from the retrospective cohort were validated prospectively in 38 additional consecutive subjects ( 23 males, 15 females; mean age $58 \pm 12$ years (range, 24 to 76 )). Twenty-two patients were included and monitored during both treatment cycles with co-infusion of $75 \mathrm{~g}$ and $50 \mathrm{~g}$ of AA, as previously reported [14]. Detailed patient characteristics are given in Table 1.

\section{Assessment of kidney function}

About 2 weeks prior to PRRT, 22 patients underwent renal scintigraphy with ${ }^{99 \mathrm{~m}}$ Tc-labelled mercaptoacetyltriglycine (MAG3) on a single head gamma camera (Signature; Siemens, Erlangen, Germany) equipped with low-energy,

Table 1 Patient characteristics

\begin{tabular}{lll}
\hline & Retrospective cohort & Prospective cohort \\
\hline Number of subjects & 38 & 38 \\
Mean age (years) & $62 \pm 14$ & $58 \pm 12$ \\
Sex & 24 males, 14 females & 23 males, \\
& & 15 females \\
Number of treatment & $3.26 \pm 2.00$ & $4.26 \pm 2.44$ \\
cycle, mean & & \\
Mean activity (GBq) & $23.91 \pm 14.67$ & $31.68 \pm 19.21$ \\
& 177 Lu-DOTATATE & 177 Lu-DOTATOC
\end{tabular}

Primary tumor

$\begin{array}{lll}\text { Pancreatic NET } & 9 & 10\end{array}$

Small bowel NET $\quad 5 \quad 4$

Ileum NET $\quad 4 \quad 6$

Cerebral tumor $\quad 4 \quad 2$

NET of unknown origin $4 \quad 2$

Gastric NET $\quad 2$

MTC 2

Caecum NET 2

Rectal NET 12

Liver NET $\quad 1 \quad 2$

Thymus NET $\quad 1$

Paraganglioma $\quad 1 \quad 1$

Lung NET $\quad 1 \quad 3$

Hemangioendothelioma

Main baseline features of the patients enrolled in the study. GBq Gigabecquerels, 177Lu-DOTATATE/-DOTATOC [1,4,7,10-tetraazacyclododecane-NN', 37 N",N"'-tetraacetic acid]-d-Phe1,Tyr3-octreotate/-toc, NET neuroendocrine tumor, MTC medullary thyroid cancer. 
high-resolution collimators. The radiotracer was prepared using a commercially available kit (MAG3, Mallinckrodt Pharmaceuticals, Neustadt an der Donau, Germany). All patients were asked to drink at least $250 \mathrm{ml}$ of water $30 \mathrm{~min}$ prior to the examination. Imaging started immediately after injection of $98 \pm 6 \mathrm{MBq}{ }^{99 \mathrm{~m}}$ Tc-MAG-3 and was performed according to national guidelines [15]. Venous blood samples were drawn from the arm contralateral to the injection site 20 and $30 \mathrm{~min}$ after tracer injection to allow for calculation of tubular extraction rates (TER). Sixteen patients underwent renal assessment by ${ }^{131}$ I-hippurate on an outpatient basis and were excluded from this part of the study.

\section{Therapy}

\section{Preparation of ${ }^{177}$ LU-DOTATATE/-TOC and PRRT}

${ }^{177} \mathrm{Lu}$-DOTATATE/-TOC was prepared with minor modifications as previously reported $[14,16]$. All patients were admitted 1 day prior to therapy to guarantee adequate hydration (1 l l saline) and were hospitalized for a total of 3 days. In the retrospective group, $37.5 \mathrm{~g}$ L-arginine hydrochloride and $37.5 \mathrm{~g}$ L-lysine hydrochloride (75 g total amount of AA, $\mathrm{pH} 7.0$ ) were intravenously administered over $4 \mathrm{~h}$ beginning $30 \mathrm{~min}$ to $1 \mathrm{~h}$ prior to PRRT. In the prospective cohort, amounts of AA infused were changed to $25 \mathrm{~g}$ of each $\mathrm{L}$-arginine and L-lysine ( $\mathrm{pH} \quad 7.0$; single-day $50 \mathrm{~g}$ protection-protocol) in order to comply with the joint IAEA, EANM, and SNMMI practical guidance [6]. No other fluids were administered. As recently published, the two different amounts of AA do not result in any difference in incidence or severity of hyperkalemia [14].

\section{Pre- and post-therapeutic blood samples}

One day before, 4 and $24 \mathrm{~h}$ after the beginning of the AA infusion, standard blood values (potassium, GFR, creatinine, BUN, sodium, phosphate, chloride [available in all patients] and lactate dehydrogenase [retrospective cohort: available in $n=36 / 38$, prospective cohort: all patients]) were assessed. Application of the tourniquet was used as short as possible to minimize false-positive potassium values due to hemolysis. Serum potassium levels were measured with the indirect ion sensitive electrode (ISE) Cobas 8000 system (Roche Diagnostics, Mannheim, Germany). All samples were screened for hemolysis. Absorbance of the diluted serum samples (dilution 1:26) was measured at 570 (primary wave length) and at $600 \mathrm{~nm}$ (secondary wave length), and hemolysis indices were calculated according to the manufacturer's instruction. Serum samples with hemolysis indices above 90 (equaling $90 \mathrm{mg} / \mathrm{dl}$ free hemoglobin) were considered to be hemolytic and excluded from further evaluation $(<3 \%$ of all samples drawn).

\section{Analysis and statistics}

Student's $t$ test was used to test for simple differences between patients with or without hyperkalemia in various blood parameters. A $p$ value of 0.05 or less was considered to be significant. Moreover, we have assumed that some blood parameters promote and some prevent hyperkalemia. Therefore, it may be possible that the human body tries to restore a homeostatic imbalance by increasing one blood parameter or decreasing another. Such relationships could not be accounted for by Student's $t$ tests. However, linear discriminant analysis makes it possible to classify patients with different conditions (e.g., hyperkalemia) on the basis of a set of predictors (e.g., multiple blood parameters). Hence, we performed multiple linear discriminant analysis to improve the prediction of severe hyperkalemia $(>6.0 \mathrm{mmol} / \mathrm{l})$ by calculating linear combinations of the relevant blood parameters. In a backward stepwise analysis, the weakest serum predictor contributing least to the prediction of severe hyperkalemia was excluded according to its $p$ value. Cutoff values for the prediction of severe hyperkalemia were determined for each formula by receiver operating characteristic (ROC) analysis [17].

Additionally, in order to test a correlation between increases of potassium and clinical factors, Pearson's correlation was performed. A $p$ value of 0.05 or less was considered to be significant.

\section{Results}

\section{AA-induced hyperkalemia (retrospective cohort)}

At baseline, mean $\mathrm{K}^{+}$levels were $4.41 \pm 0.37 \mathrm{mmol} / \mathrm{l} .4 \mathrm{~h}$ after PRRT, mean serum potassium level increased to $5.90 \pm 0.80 \mathrm{mmol} / \mathrm{l}$ with values $\geq 5.0 \mathrm{mmol} / \mathrm{l}$ in $36 / 38$ subjects (94.7\%; 17/36 subjects with history of potassiumsparing anti-hypertensive medications and long-term use of NSAIDs). Thirteen of those 36 patients (36.1\%) experienced severe hyperkalemia $>6.0 \mathrm{mmol} / \mathrm{l}$. Only $2 / 38$ subjects did not show elevated $\mathrm{K}^{+}$levels.

Five of the 13 subjects (38.5\%, 2/5 with diabetes) with severe hyperkalemia became symptomatic with palpitations and general malaise. In all five patients, ECG revealed flattened $P$ waves and high peaked $T$ waves with peak serum potassium levels of $8.5 \mathrm{mmol} /$ 1. These patients required immediate therapy to lower serum potassium concentrations. We effectively administered 10 units of regular insulin in $500 \mathrm{ml}$ of $10 \%$ dextrose over $60 \mathrm{~min}$.

At $24 \mathrm{~h}, \mathrm{~K}^{+}$levels had almost returned to baseline levels (mean $4.55 \pm 0.42 \mathrm{mmol} / \mathrm{l}, p>0.05$ compared to baseline; $p<0.001$ compared to $4 \mathrm{~h}$ ) in $32 / 38(84.2 \%)$ subjects. Six of the 38 patients (15.8\%) still demonstrated slightly elevated potassium levels with a maximum of $5.5 \mathrm{mmol} / \mathrm{l}$ (Figure 1; Table 2). 


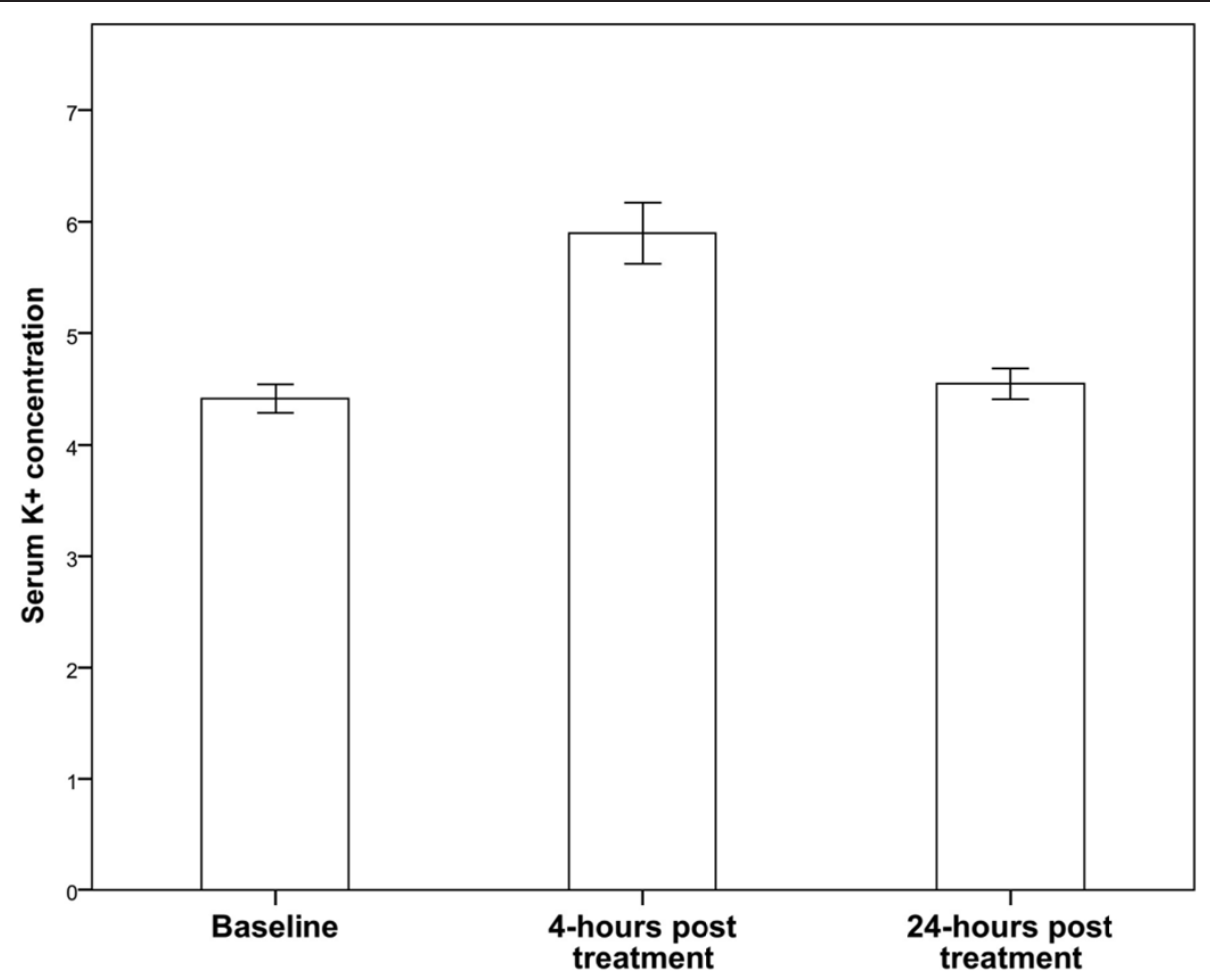

Figure 1 Serum potassium concentrations ( $\mathrm{mmol} / \mathrm{l}$ ) at baseline, 4 and $24 \mathrm{~h}$ after PRRT (retrospective cohort, $n=38$ ).

\section{Correlation of hyperkalemia to kidney function and clinical factors (retrospective cohort)}

All patients presented with normal kidney function according to ${ }^{99 \mathrm{~m}}$ Tc-MAG3 scintigraphy with a mean TER of $231 \pm 47 \mathrm{ml} / \mathrm{min} / 1.73 \mathrm{~m}^{2}$. The pre-therapeutic TER did not correlate with the post-therapeutic potassium level $(r=-0.120)$ and was unable to predict incidence or severity of hyperkalemia. No cutoff value of minimum glomerular filtration rate excluding severe hyperkalemia could be derived from serum blood samples $(r=-0.055$, $p=0.74)$.
Additional serum parameters (potassium, BUN, sodium, phosphate, chloride, LDH) were analyzed (Table 2). Out of all variables investigated, a pre-therapeutic BUN cutoff value of $>28 \mathrm{mg} / \mathrm{dl}$ (defined by ROC analysis) had a sensitivity of $84.6 \%$ and a specificity of $60 \%$ in predicting severe hyperkalemia $\geq 6.0 \mathrm{mmol} / 1$ (area under the curve, AUC $=0.75)$. No clinical characteristic including patient age, cumulative dose, number of treatment cycles, potentially interfering medications, or clinical risk factors correlated with the incidence or severity of post-treatment hyperkalemia (Additional file 1: Table S1).

Table 2 Biochemical parameters of patients at baseline, 4 and $24 \mathrm{~h}$ after PRRT

\begin{tabular}{lllll}
\hline & Normal range & Baseline & $\mathbf{4} \mathbf{h}$ & $\mathbf{2 4} \mathbf{h}$ \\
\hline Potassium $(\mathrm{mmo} / \mathrm{l})$ & 3.5 to 5.0 & $4.41 \pm 0.37$ & $5.9 \pm 0.8$ & $4.55 \pm 0.42$ \\
GFR $(\mathrm{CKD}-\mathrm{EPI})\left(\mathrm{ml} / \mathrm{min} / 1.73 \mathrm{~m}^{2}\right)$ & - & $82.79 \pm 25.49$ & $80 \pm 23.81$ & $81.22 \pm 26.34$ \\
Creatinine $(\mathrm{mg} / \mathrm{dl})$ & 0 to 0.95 & $0.93 \pm 0.28$ & $0.95 \pm 0.26$ & $0.97 \pm 0.29$ \\
BUN $(\mathrm{mg} / \mathrm{dl})$ & 10 to 50 & $33.32 \pm 12.89$ & $42.38 \pm 12.22$ & $36.95 \pm 14.07$ \\
Sodium $(\mathrm{mmo} / \mathrm{l})$ & 135 to 145 & $138.79 \pm 3.03$ & $133.63 \pm 3.11$ & $137.42 \pm 14.07$ \\
Phosphate $(\mathrm{mmol} / \mathrm{l})$ & 0.87 to 1.45 & $1.07 \pm 0.19$ & $0.84 \pm 0.16$ & $1.21 \pm 0.22$ \\
Chloride $(\mathrm{mmol} / \mathrm{l})$ & 94 to 110 & $99.75 \pm 4.3$ & $107.84 \pm 3.7$ & $105.32 \pm 4.15$ \\
LDH $(\mathrm{U} / \mathrm{l})$ & $\leq 250$ & $218.87 \pm 59.98$ & $220.38 \pm 55.2$ & $219.36 \pm 45.21$ \\
\hline
\end{tabular}

Biochemical parameters of patients at baseline, 4 and $24 \mathrm{~h}$ after PRRT (retrospective cohort; LDH, $n=36 / 38$; all other parameters, $n=38$ ). Values are given as mean \pm SD. GFR glomerular filtration rate, CKD-EPI Chronic Kidney Disease Epidemiology Collaboration, BUN blood urea nitrogen, LDH lactate dehydrogenase, $\mathrm{mmol} / \mathrm{l}$ millimoles per litre, $\mathrm{ml} / \mathrm{min}$ millilitres per minute, $\mathrm{mg} / \mathrm{dl}$ milligrams per decilitre, $\mathrm{U} / \mathrm{l}$ units per litre. 

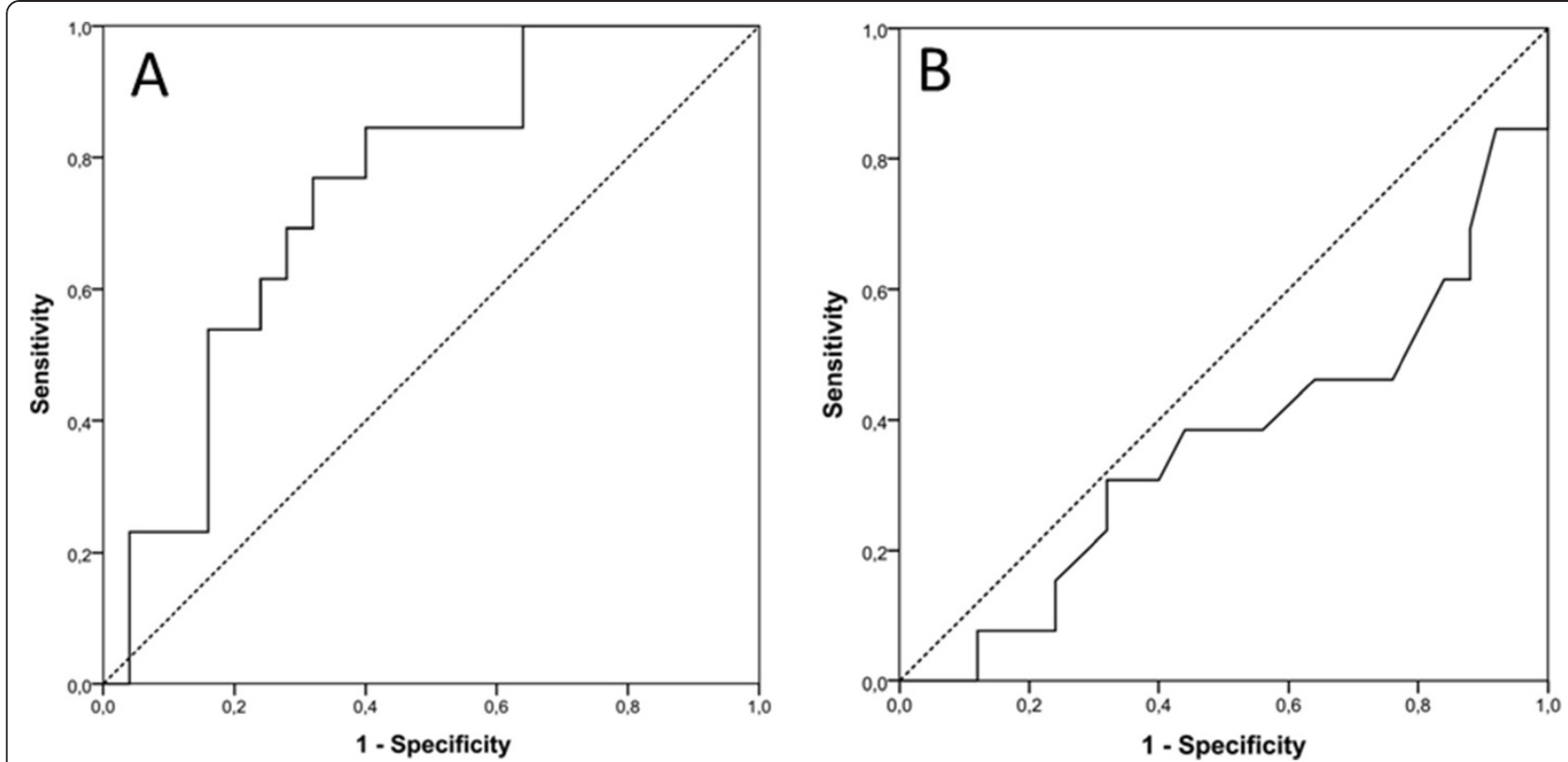

Figure 2 Receiver operating characteristic curves for blood urea nitrogen and phosphate. Receiver operating characteristic (ROC) curves for (A) serum blood urea nitrogen (BUN) and (B) phosphate for the prediction of severe hyperkalemia (>6.0 mmol/I) after peptide receptor radionuclide therapy (retrospective cohort, $n=38$ ). BUN demonstrates some value as a single, simply achievable parameter (area under the curve $[A \cup C]=0.75)$ whereas serum phosphate is not a suitable predictor $(A \cup C=0.37$ ).

We also investigated changes to the baseline to $4-\mathrm{h}$ post-therapy initiation. Changes in serum BUN tended to positively correlate with hyperkalemia $(r=0.313 ; p<0.056)$ (Figure 2A). A significant negative correlation between increase in serum potassium and phosphate was observed $(r=-0.444 ; p<0.005)$. However, this parameter did not predict severe hyperkalemia $(>6.0 \mathrm{mmol} / \mathrm{l}) \quad(\mathrm{AUC}=0.37)$ (Figure 2B).

\section{Calculation of formulas for hyperkalemia-risk-assessment (retrospective cohort)}

In order to improve estimation of the risk of severe hyperkalemia, several formulas were generated based on an arbitrarily set cutoff potassium level $>6.0 \mathrm{mmol} / \mathrm{l}$ and different serum parameters. Three of these formulas showed superior results in retrospective evaluation.

The parameters used in the formulas are the pretherapeutic plasma concentrations of potassium $\left(\mathrm{K}^{+}\right.$, in $\mathrm{mmol} / \mathrm{l}$, available in $38 / 38$ patients), sodium $\left(\mathrm{Na}^{+}\right.$, in $\mathrm{mmol} / \mathrm{l}, n=38 / 38$ ), phosphate $\left(\mathrm{PO}^{2-}{ }^{2-}\right.$ in $\mathrm{mmol} / \mathrm{l}$, $n=38 / 38$ ), creatinine (Crea, in $\mathrm{mg} / \mathrm{dl}, n=38 / 38$ ), blood urea nitrogen (BUN, in $\mathrm{mg} / \mathrm{dl}, n=38 / 38$ ), lactate dehydrogenase ( $\mathrm{LDH}$, in $\mathrm{U} / \mathrm{l}, n=36 / 38$ ), as well as the glomerular filtration rate (GFR, in $\mathrm{ml} /$ $\left.\min / 1.73 \mathrm{~m}^{2}, n=38 / 38\right)$.
The first formula included all seven parameters:

Formula $1(n=36)$ :

$$
\begin{aligned}
X= & 1.6 \cdot \mathrm{K}^{+}+0.055 \cdot \mathrm{BUN}+0.004 \cdot \mathrm{LDH}-\left(0.243 \cdot \mathrm{Na}^{+}\right. \\
& \left.+3.922 \cdot \mathrm{PO}^{2-}+0.009 \cdot \mathrm{GFR}+1.358 \cdot \text { Crea }\right)
\end{aligned}
$$

Defining the cutoff as $X \geq-29.909$ resulted in a sensitivity of $84.6 \%$ and a specificity of $82.6 \%$, respectively (AUC $=0.92$, accuracy $=83.3 \%$ ).

GFR and creatinine were omitted in Formula 2:

Formula $2(n=36)$ :

$$
\begin{aligned}
X= & 1.742 \cdot \mathrm{K}^{+}+0.045 \cdot \mathrm{BUN} \\
& +0.003 \cdot \mathrm{LDH}-\left(0.252 \cdot \mathrm{Na}^{+}+3.927 \cdot \mathrm{PO}^{2-}\right)
\end{aligned}
$$

Based on an AUC of 0.90 for Formula 2, the optimal cutoff value was defined as $X \geq-29.226$ with a sensitivity of $92.3 \%$ and a specificity of $82.6 \%$ (accuracy $=86.1 \%$ ).

Excluding further two parameters (BUN, LDH) while introducing creatinine led to Formula 3:

Formula $3(n=38)$ :

$$
X=2.046 \cdot \mathrm{K}^{+}+1.053 \cdot \mathrm{Crea}-\left(0.305 \cdot \mathrm{Na}^{+}+4.356 \cdot \mathrm{PO}^{2-}\right)
$$

ROC analysis revealed an AUC of 0.90. A cutoff value of $X \geq-37.035$ resulted in a sensitivity of $92.3 \%$ and a specificity of $72.0 \%$ in excluding severe hyperkalemia 

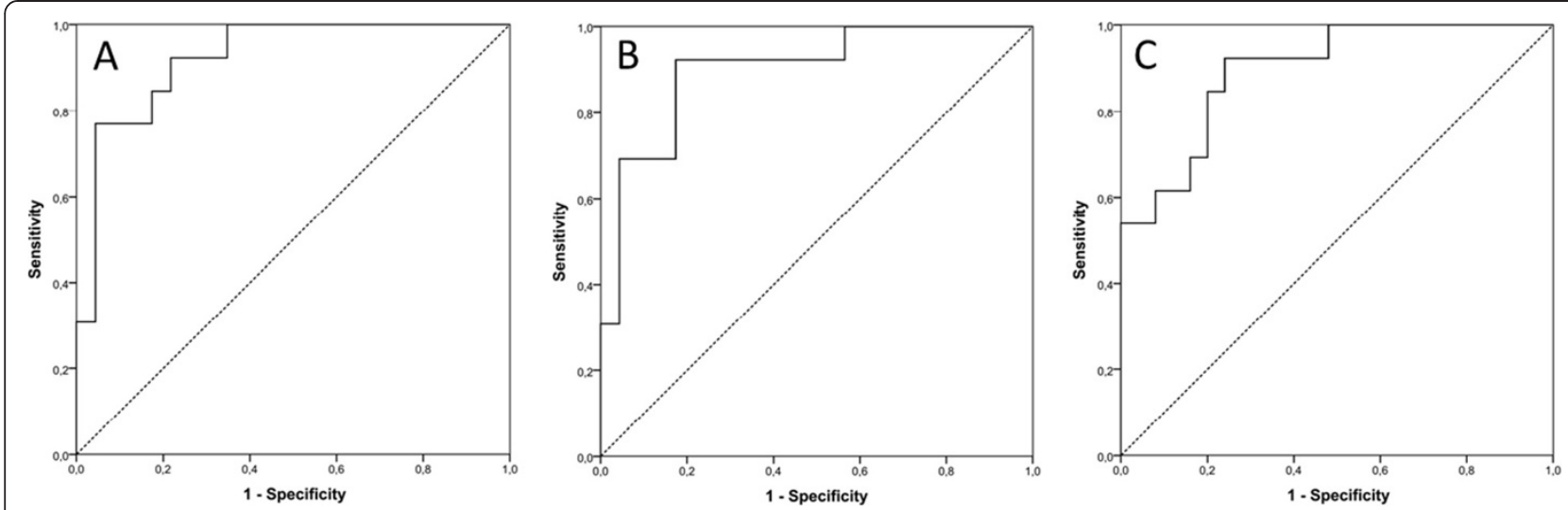

Figure 3 Receiver operating characteristic curves for the prediction of severe hyperkalemia (>6.0 mmol/l). Receiver operating characteristic (ROC) curves for the three formulas computed for the prediction of severe hyperkalemia (>6.0 mmol/l) with (A) seven-, (B) five-, and (C) four-serum parameters. Pre-therapeutic plasma concentrations of potassium, sodium, phosphate, creatinine, blood urea nitrogen, lactate dehydrogenase, as well as the glomerular filtration rate were included. All formulas show excellent areas under the curve ranging from 0.90 to 0.92 .

(accuracy $=78.9 \%)$. The respective ROC curves are given in Figure 3.

\section{Validation of formulas for hyperkalemia-risk-assessment (prospective cohort)}

In a prospective setup, additional 38 patients were enrolled to validate the formulas: none of the patients suffered from hyperkalemia prior to PRRT with mean potassium levels of $4.34 \pm 0.39 \mathrm{mmol} / \mathrm{l}$. $4 \mathrm{~h}$ after PRRT, mean $\mathrm{K}^{+}$level was $5.69 \pm 0.61 \mathrm{mmol} / \mathrm{l}$ with values $\geq 5.0 \mathrm{mmol} / \mathrm{l}$ in $28 / 38$ subjects $(73.6 \%$; $15 / 28$ subjects with history of potassium-sparing anti-hypertensive medications and long-term use of NSAIDs). Nine of the 38 patients $(23.7 \%)$ experienced severe hyperkalemia $>6.0 \mathrm{mmol} / \mathrm{l}$, and three patients became symptomatic with palpitations and ECG changes. After $24 \mathrm{~h}, \mathrm{~K}^{+}$levels returned to baseline levels (mean $4.53 \pm 0.37 \mathrm{mmol} / \mathrm{l}$ ).

The three formulas were validated using the cutoff values mentioned above. Formula 1 showed a sensitivity of $77.8 \%$ and a specificity of $79.3 \%$ (accuracy $=78.9 \%$ ) and Formula 2 had a sensitivity of $88.9 \%$ and a specificity of $79.3 \%$ (accuracy $=81.6 \%$ ). Formula 3, including four blood values, could predict severe hyperkalemia $>6.0 \mathrm{mmol} / \mathrm{l}$ with a sensitivity of $66.7 \%$ and a specificity of $82.8 \%$ (accuracy $=78.9 \%)$.

An overview over the results of the three formulas is given in Table 3 (retrospective and prospective cohort).

\section{Discussion}

The high incidence of PRRT-related hyperkalemia in patients treated for NET has been recently reported [13]. The aim of this study was to identify pre-therapeutic parameters for prediction of AA-induced hyperkalemia in patients undergoing PRRT. Combinations of serum parameters were retrospectively identified, and their predictive value was prospectively evaluated. No single serum parameter could reliably predict the occurrence of hyperkalemia. In the prospective validation cohort, a formula including five routine biochemical serum parameters (potassium, BUN, sodium, phosphate, LDH; Formula 2) correctly identified 8 of 9 patients with (sensitivity: $88.9 \%$ ) and 23 of 29 patients without hyperkalemia $>6.0 \mathrm{mmol} / \mathrm{l}$ (specificity: $79.3 \%$ ); resulting in an accuracy of 31 of 38 (81.6\%).

Showing the highest sensitivity and a false-negative rate of $11.1 \%$, thus really identifying patients suffering from clinically relevant hyperkalemia, Formula 2 seems to be the most promising. Considering a potentially life-threatening side-effect, this approach might be a quite valuable tool for the clinical physician planning PRRT.

Arginine monohydrochloride and lysine monohydrochloride, both cationic amino acids, are known to cause transient hyperkalemia by driving potassium from the intracellular to the extracellular compartment

Table 3 Overview of results (retrospective and prospective cohort)

\begin{tabular}{|c|c|c|c|c|c|c|}
\hline \multirow[b]{2}{*}{ Formula } & \multicolumn{3}{|c|}{ Retrospective cohort (75 g AA) } & \multicolumn{3}{|c|}{ Prospective cohort (50 g AA) } \\
\hline & Sensitivity & Specificity & Accuracy & Sensitivity & Specificity & Accuracy \\
\hline 1 & $84.6(11 / 13)$ & $82.6(19 / 23)$ & $83.3(30 / 36)$ & $77.8(7 / 9)$ & $79.3(23 / 29)$ & $78.9(30 / 38)$ \\
\hline 2 & $92.3(12 / 13)$ & $82.6(19 / 23)$ & $86.1(31 / 36)$ & $88.9(8 / 9)$ & $79.3(23 / 29)$ & $81.6(31 / 38)$ \\
\hline 3 & $92.3(12 / 13)$ & $72.0(18 / 25)$ & $78.9(30 / 38)$ & $66.7(6 / 9)$ & $82.8(24 / 29)$ & $78.9(30 / 38)$ \\
\hline
\end{tabular}

Overview of results (retrospective cohort, Formulas 1 and 2: $n=36$; Formula 3: $n=38$, left; prospective cohort, all formulas: $n=38$, right). Sensitivity, specificity, and accuracy are given in percentage. Numbers in brackets indicate patient numbers. $A A=$ amino acid solution. 
$[18,19]$. In our cohort, hyperkalemia $(>5.0 \mathrm{mmol} / \mathrm{l})$ was induced in almost every patient and $\mathrm{K}^{+}$levels rose by $33.8 \%$. This finding is in line with previous results $[11,13,20]$. Thus, close monitoring of patients after PRRT and evaluation of serum potassium levels should be mandatory as more than $35 \%$ of subjects $(13 / 36)$ experienced rises in $\mathrm{K}^{+}$to $>6.0 \mathrm{mmol} / \mathrm{l}$. Five patients became symptomatic and required potassium-lowering interventions (e.g. insulin with glucose). Of note, two of those patients suffered from diabetes ( $40 \%$ of all diabetic patients in this cohort), a sub-cohort maybe especially prone to post-therapeutic hyperkalemia due to reduced sensitivity to endogenous insulin. An earlier study has already reported on this pathophysiologic mechanism [21]. In our study, no significant correlation between diabetes and incidence or severity of increases in potassium could be detected; however, this might be related to small sample sizes.

To prevent potential complications, we aimed to identify predictors for severe hyperkalemia $>6.0 \mathrm{mmol} / \mathrm{l}$ to help the clinician in identifying patients at risk. The only factor with limited predictive value was an increased serum BUN. A cutoff value of $>28 \mathrm{mg} / \mathrm{dl}$ provided a sensitivity of $84.6 \%$ but a rather low specificity of $60.0 \%$ in excluding severe hyperkalemia $>6.0 \mathrm{mmol} / \mathrm{l}(\mathrm{AUC}=0.75)$. However, since emphasis should be put on high sensitivity, this marker is suggested as simple first test in evaluating potentially severe hyperkalemia.

In a more sophisticated approach, a combination of biochemical markers which are all affected by PRRT was used to derive three different formulas for predicting the risk of AA-induced hyperkalemia. The four-to-seven parameters considered by these formulas can be obtained from one blood sample. All formulas showed excellent areas under the curve in retrospective ROC analysis (range, 0.90 to 0.92 ).

In order to allow broad applicability, accuracy was prospectively investigated. In this cohort, the formula taking into account five serum parameters (potassium, creatinine, sodium, LDH and phosphate; Formula 2) demonstrated again both excellent sensitivity (88.9\%) and good specificity (79.3\%).

The following limitations have to be considered: in order to strengthen the statistical power and the prognostic significance of our findings, further studies with larger patient cohorts need to be performed. Secondly, almost more than half of the patients were included and monitored during both retrospective and prospective treatment cycles with co-infusion of 75 or $50 \mathrm{~g}$ of AA. Moreover, the amount of AA routinely administered was reduced from 75 to $50 \mathrm{~g}$ to comply with the latest joint IAEA, EANM, and SNMMI practical guidance in October 2013 [6]. Thus, the retrospectively calculated formulas were derived administering $75 \mathrm{~g}$ AA whereas in the prospective part these findings were validated administering $50 \mathrm{~g}$ AA. However, in our intra-individual comparison of both protocols, incidence and severity of hyperkalemia do not significantly vary between 75 and $50 \mathrm{~g}$ AA, respectively [14]. Due to this finding of our previous study, a broad applicability in clinical routine is independent from AA dosing (75 or $50 \mathrm{~g}$, respectively). On a final note, different radiolabelled compounds were used. In the prospective cohort, DOTATATE was replaced by DOTATOC. Esser and colleagues reported that therapy with ${ }^{177} \mathrm{Lu}$-octreotate is preferable due to its higher tumor residence time. However, this research group did not report on incidence of hyperkalemia after administering both protocols [22].

In conclusion, the high incidence of hyperkalemia in almost all patients undergoing PRRT requires a high degree of clinical attention. Serum blood sampling after treatment is mandatory, given that more than $20 \%$ of patients experience a potentially life-threatening rise in $\mathrm{K}^{+}$values to $>6.0 \mathrm{mmol} / \mathrm{l}$. Early identification of subjects at risk who might require immediate therapeutic intervention would be of great value. To the best of our knowledge, this is the first attempt to derive a robust estimate of the risk of AA-induced hyperkalemia in patients scheduled for PRRT. With an accuracy of $81.6 \%$ and a low false-negative rate, we established a reliable prospectively validated formula assessed by one simple blood collection selecting 'high risk' patients being vulnerable for severe hyperkalemia. In general, this formula might be a valuable tool for the clinical physician planning PRRT and was recently implemented into our clinical routine.

\section{Conclusions}

No single serum-based factor can reliably predict posttherapeutic increases in potassium to values $>6.0 \mathrm{mmol} / \mathrm{l}$ after PRRT. Our data suggest that prediction of severe hyperkalemia using a combination of routine serum parameters prior to PRRT including potassium, creatinine, sodium, LDH, and phosphate identified 'high risk' patients with an accuracy of $81.6 \%$. We therefore conclude that this formula is a valuable tool for identifying patients prone to develop PRRT-associated severe hyperkalemia.

\section{Additional file}

Additional file 1: Correlation of clinical factors with post-treatment increases in serum potassium.

\section{Competing interests}

The authors declare that they have no competing interests. 


\section{Authors' contributions}

$C L, R A W, K L, A n S, A K B, M A A$, and $K H$ conceived the study. CB and AIS acquired the data. $C L, R A W, H H$, and DOM analyzed and interpreted the data. $C L, R A W, C B$, and AlS were involved in the drafting of the manuscript; and $K L, A n S, A K B, R A B, C B$, and $K H$ revised it critically for important intellectual content. All authors gave final approval of the version to be published and agree to be accountable for all aspects of the work in ensuring that questions related to the accuracy or integrity of any part of the work are appropriately investigated and resolved.

\section{Acknowledgements}

We thank Irene Grelle, Hannelore Jahn, Susanne Hirsch, and Karin Knorr (members of the nuclear medicine clinical laboratory) for their support and assistance.

\section{Author details}

'Department of Nuclear Medicine, University Hospital Würzburg, Oberdürrbacher Str. 6, Würzburg 97080, Germany. ${ }^{2}$ Institute of Psychology, University of Innsbruck, Innsbruck, Austria. Institute of Clinical Chemistry, University Hospital Würzburg, Oberdürrbacher Str. 6, Würzburg 97080, Germany. ${ }^{4}$ Ahmanson Translational Imaging Division, Department of Molecular and Medical Pharmacology, David Geffen School of Medicine at UCLA, 10833 Le Conte Avenue, Los Angeles, CA 90095, USA. ${ }^{5}$ Department of Nuclear Medicine, University Hospital Bonn, Sigmund-Freud-Straße 25, Bonn 53127, Germany.

Received: 8 November 2014 Accepted: 1 December 2014

Published online: 24 December 2014

\section{References}

1. Modlin IM, Oberg K, Chung DC, Jensen RT, de Herder WW, Thakker RV, Caplin M, Delle Fave G, Kaltsas GA, Krenning EP, Moss SF, Nilsson O, Rindi G, Salazar R, Ruszniewski P, Sundin A: Gastroenteropancreatic neuroendocrine tumours. Lancet Oncol 2008, 9:61-72

2. Waser B, Tamma ML, Cescato R, Maecke HR, Reubi JC: Highly efficient in vivo agonist-induced internalization of sst2 receptors in somatostatin target tissues. J Nucl Med 2009, 50:936-941.

3. Bergsma H, van Vliet El, Teunissen JJ, Kam BL, de Herder WW, Peeters RP Krenning EP, Kwekkeboom DJ: Peptide receptor radionuclide therapy (PRRT) for GEP-NETs. Best Pract Res Clin Gastroenterol 2012, 26:867-881.

4. Imhof A, Brunner P, Marincek N, Briel M, Schindler C, Rasch H, Macke HR, Rochlitz C, Muller-Brand J, Walter MA: Response, survival, and long-term toxicity after therapy with the radiolabeled somatostatin analogue [90Y-DOTA]-TOC in metastasized neuroendocrine cancers. J Clin Oncol 2011, 29:2416-2423.

5. Valkema R, Pauwels SA, Kvols LK, Kwekkeboom DJ, Jamar F, de Jong M Barone R, Walrand S, Kooij PP, Bakker WH, Lasher J, Krenning EP: Long-term follow-up of renal function after peptide receptor radiation therapy with (90)Y-DOTA(0), Tyr(3)-octreotide and (177)Lu-DOTA(0), Tyr(3)-octreotate. J Nucl Med 2005, 46:83-91.

6. Bodei L, Mueller-Brand J, Baum RP, Pavel ME, Horsch D, O'Dorisio MS, O'Dorisio TM, Howe JR, Cremonesi M, Kwekkeboom DJ, Zaknun JJ: The joint IAEA, EANM, and SNMMI practical guidance on peptide receptor radionuclide therapy (PRRNT) in neuroendocrine tumours. Eur I Nucl Med Mol Imaging 2013, 40:800-816.

7. de Jong $M$, Krenning E: New advances in peptide receptor radionuclide therapy. J Nucl Med 2002, 43:617-620.

8. Jamar F, Barone R, Mathieu I, Walrand S, Labar D, Carlier P, de Camps J, Schran H, Chen T, Smith MC, Bouterfa H, Valkema R, Krenning EP, Kvols LK, Pauwels S: 86Y-DOTA0)-D-Phe1-Tyr3-octreotide (SMT487)-a phase 1 clinical study: pharmacokinetics, biodistribution and renal protective effect of different regimens of amino acid co-infusion. Eur J Nucl Med Mol Imaging 2003, 30:510-518.

9. Bodei L, Cremonesi M, Grana C, Rocca P, Bartolomei M, Chinol M, Paganelli G: Receptor radionuclide therapy with 90Y-[DOTA]0-Tyr3-octreotide (90Y-DOTATOC) in neuroendocrine tumours. Eur J NuCl Med Mol Imaging 2004, 31:1038-1046.

10. Werner RA, Bluemel C, Allen-Auerbach MS, Higuchi T, Herrmann K: ${ }^{68}$ Gallium- and ${ }^{90}$ Yttrium- $/{ }^{177}$ Lutetium: "theranostic twins" for diagnosis and treatment of NETs. Ann NuCl Med 2014. doi:10.1007/s12149-014-0898-6
11. Rolleman EJ, Valkema R, de Jong M, Kooij PP, Krenning EP: Safe and effective inhibition of renal uptake of radiolabelled octreotide by a combination of lysine and arginine. Eur J Nucl Med Mol Imaging 2003, 30:9-15.

12. Bernard BF, Krenning EP, Breeman WA, Rolleman EJ, Bakker WH, Visser TJ, Macke $\mathrm{H}$, de Jong M: D-lysine reduction of indium-111 octreotide and yttrium-90 octreotide renal uptake. J Nucl Med 1997, 38:1929-1933.

13. Giovacchini $G$, Nicolas $G$, Freidank $H$, Mindt $T L$, Forrer F: Effect of amino acid infusion on potassium serum levels in neuroendocrine tumour patients treated with targeted radiopeptide therapy. Eur J Nucl Med Mol Imaging 2011, 38:1675-1682.

14. Lapa C, Werner RA, Bluemel C, Lückerath K, Schirbel A, Strate A, Buck AK Herrmann $\mathrm{K}$ : Influence of the amount of co-infused amino acids on post-therapeutic potassium levels in peptide receptor radionuclide therapy. EJNMMI Res 2014, 4:46

15. Hahn K, Franzius C: DGN-Handlungsempfehlung (S1-Leitlinie) Nierenfunktionsszintigraphie mit und ohne Furosemidbelastung be Kindern und Erwachsenen. AWMF-Registernummer: 031-042. 04/2013.

16. Kwekkeboom DJ, Bakker WH, Kooij PP, Konijnenberg MW, Srinivasan A, Erion JL, Schmidt MA, Bugaj JL, de Jong M, Krenning EP: [177Lu-DOTAOTyr3] octreotate: comparison with [111ln-DTPAo]octreotide in patients. Eur J NuCl Med 2001, 28:1319-1325.

17. Zou KH, O'Malley AJ, Mauri L: Receiver-operating characteristic analysis for evaluating diagnostic tests and predictive models. Circulation 2007, 115:654-657.

18. Levinsky NG, Tyson I, Miller RB, Relman AS: The relation between amino acids and potassium in isolated rat muscle. J Clin Invest 1962, 41:480-487.

19. Dickerman HW, Walker WG: Effect of cationic amino acid infusion on potassium metabolism in vivo. Am J Physio/ 1964, 206:403-408.

20. Barone R, Pauwels S, De Camps J, Krenning EP, Kvols LK, Smith MC, Bouterfa H, Devuyst O, Jamar F: Metabolic effects of amino acid solutions infused for renal protection during therapy with radiolabelled somatostatin analogues. Nephrol Dial Transplant 2004, 19:2275-2281.

21. Massara F, Cagliero E, Bisbocci D, Passarino G, Carta Q, Molinatti GM: The risk of pronounced hyperkalaemia after arginine infusion in the diabetic subject. Diabetes Metab 1981, 7:149-153.

22. Esser JP, Krenning EP, Teunissen J, Kooij PP, van Gameren AL, Bakker WH, Kwekkeboom DJ: Comparison of [(177)Lu-DOTA(0), Tyr(3)]octreotate and [(177)Lu-DOTA(0), Tyr(3)]octreotide: which peptide is preferable for PRRT? Eur J Nucl Med Mol Imaging 2006, 33:1346-1351.

\section{Submit your manuscript to a SpringerOpen ${ }^{\circ}$ journal and benefit from:}

- Convenient online submission

- Rigorous peer review

- Immediate publication on acceptance

- Open access: articles freely available online

- High visibility within the field

- Retaining the copyright to your article

Submit your next manuscript at $>$ springeropen.com 